\title{
Collapse and Measures of Consciousness
}

\author{
Adrian Kent ${ }^{1,2}$ (D) \\ Received: 31 January 2021 / Accepted: 6 May 2021 / Published online: 25 May 2021 \\ () The Author(s) 2021
}

\begin{abstract}
There has been an upsurge of interest lately in developing Wigner's hypothesis that conscious observation causes collapse by exploring dynamical collapse models in which some purportedly quantifiable aspect(s) of consciousness resist superposition. Kremnizer-Ranchin, Chalmers-McQueen and Okon-Sebastián have explored the idea that collapse may be associated with a numerical measure of consciousness. More recently, Chalmers-McQueen have argued that any single measure is inadequate because it will allow superpositions of distinct states of equal consciousness measure to persist. They suggest a satisfactory model needs to associate collapse with a set of measures quantifying aspects of consciousness, such as the "Q-shapes" defined by Tononi et al. in their "integrated information theory" (IIT) of consciousness. I argue here that Chalmers-McQueen's argument against associating a single measure with collapse requires a precise symmetry between brain states associated with different experiences and thus does not apply to the only case where we have strong intuitions, namely human (or other terrestrial biological) observers. In defence of Chalmers-McQueen's stance, it might be argued that idealized artificial information processing networks could display such symmetries. However, I argue that the most natural form of any theory (such as IIT) that postulates a map from network states to mind states is one that assigns identical mind states to isomorphic network states (as IIT does). This suggests that, if such a map exists, no familiar components of mind states, such as viewing different colours, or experiencing pleasure or pain, are likely to be related by symmetries.
\end{abstract}

Keywords Wigner hypothesis · Quantum mechanics · Wave function collapse · Consciousness $\cdot$ Integrated information theory $\cdot$ Symmetry

Adrian Kent

A.P.A.Kent@damtp.cam.ac.uk

1 Centre for Quantum Information and Foundations, DAMTP, Centre for Mathematical Sciences, University of Cambridge, Wilberforce Road, Cambridge CB3 0WA, U.K.

2 Perimeter Institute for Theoretical Physics, 31 Caroline Street North, Waterloo, ON N2L 2Y5, Canada 


\section{Introduction}

The hypothesis that wave function collapse is an objective process, caused by conscious observation, is widely attributed to Wigner [1]; a more detailed history, starting with discussions by London-Bauer and von Neumann, is given by Chalmers and McQueen [2, 3].

It has recently been revived by proposals [2-8] aimed at defining precise dynamical theories that combine ideas for proposed objective quantifications of aspects of consciousness - in particular Tononi et al.'s “integrated information theory” (IIT) [9] and related ideas - with objective dynamical collapse models. Dynamical collapse models $[10,11]$ propose parametrised stochastic differential equations that approximately reproduce pure unitary quantum evolution in one regime and approximately reproduce the mathematical effect of the projection postulate, which characterises the effects of measuring a quantum state, in another regime. With appropriate choices of equations and parameters, they can imply all the successful predictions of quantum theory in interference and other experiments to date, while making testably different predictions in possible (although generally technologically challenging) future experiments. They thus are testable candidate solutions for the quantum measurement problem.

The various proposals [2-8] have different features and are based on different assumptions. It seems fair to say that all of them are projects in progress, and in each case it remains open to question whether a fully defined and generally viable dynamical collapse model with all the desired features will emerge, even in the nonrelativistic limit. Chalmers and McQueen [2], whose work is our main focus here, propose to model IIT (or some other such classical model of consciousness) within quantum theory by quasiclassical operators. On the face of it this seems viable in regions, such as our own environment, where physics can be described quasiclassically. It seems hard, though, to extend it in a way that would combine naturally with a quantum theory of matter to produce a fundamental theory applicable in all domains.

Many other fundamental questions can be raised about the motivations for and viability of this programme and of those it subsumes; many of these are carefully reviewed in Ref. [2] and in works cited therein. All versions of quantum theory with explicit collapse are somewhat ad hoc; relativistic collapse models are hard to define; Everettians (see e.g. [12]) would argue that collapse models are unnecessary. Many (e.g. [13]) argue that everything about consciousness is completely explained by known science; on this view, it seems no more plausible that it plays a role in fundamental physics than that any other biological phenomenon does. In the other camp, many who see motivation for a theory of consciousness criticise IIT as ad hoc, under-defined and having implausible implications (see e.g. [14-17]). Still, as Chalmers-McQueen note, the idea that consciousness causes collapse has some motivation, was taken seriously by some of the pioneers of quantum theory, and, given that there is no consensus solution to the quantum measurement problem, 
seems worth keeping on the table for now and examining more carefully. ${ }^{1}$ As they also note, their proposals and arguments should apply to a wide range of quantitative theories of consciousness; we may take IIT as a placeholder that illustrates some of the issues that would also arise with possibly more satisfactory proposals.

For the sake of discussion, let us accept this view. This note focuses on one key question that Chalmers and McQueen discuss: could a satisfactory conscious-collapse model be defined in which the only quantity relevant to the collapse rate is some proposed quantitative measure of consciousness, such as the $\Phi$ measure of IIT? The idea here is that " $\Phi$ resists superposition and superpositions of $\Phi$ trigger collapse". That is, a superposition of two or more quantum states that include a possibly conscious subsystem, in which the superposition components contain states of that subsystem that have different values of $\Phi$, will undergo collapse at a rate that depends on the details of the model and the relevant $\Phi$ values. Moreover, this is the only cause of collapse, or at least the only cause generally relevant in situations where biological organisms or classical or quantum computers observe-and, in the absence of collapse, would thus themselves enter and remain in-quantum superpositions.

Chalmers and McQueen argue that [3] “... this view faces a fatal problem.” Their concern is that it fails to suppress superpositions of qualitatively distinct but equal- $\Phi$ conscious states. For example, they consider [18] an experiment in which a conscious subject observes a screen that can display blue or green in a dark isolated room. If the screen is put into a superposition of displaying both, then the subject will be put into a superposition of both experiences. They argue that there is no reason to assume that these experiences differ in their $\Phi$ value. If so, then there is no superposition of states with distinct $\Phi$ values, and so a $\Phi$-collapse model will leave the superposition uncollapsed.

\section{Human Observers}

It seems to me there is good reason to assume that the relevant experiences differ in their $\Phi$ value, at least for terrestrial animals - the only subjects we have good reasons to believe to be conscious. Brains and central nervous systems are messy, noisy, imperfect networks. It seems very unlikely that, for any given subject, the blue and green screens would excite exactly the same numbers and rates of firing for retinal cells. Even if they did, it seems very unlikely that the subsequent chains of firings would be along isomorphic neural network paths, at the same sets of times. And even if this were true in some impossibly refined meditative state, in which no other brain processes relevant to consciousness interacted in any way with the pure perception of blue or green, it seems very unlikely that this state could stably persist for any length of time. The reason is that introspection and neural network models both suggest we build up models of the world by association and memory. When we see (say) blue, our dominant perception is of the colour, but it is tinged with

\footnotetext{
1 This might produce compelling arguments against it; that too would be valuable.
} 
memories of landscapes, animals and art, with past experiences, and with emotional associations with all of these. These may flicker in and out of consciousness, but are hard to suppress completely. And even if we can fleetingly manage to focus on the pure sensation of blueness, our unconscious neural processing is still working on a network that encodes the learned associations, and our $\Phi$ value depends (in general) on all these details. Our associations with and memories of green are different, and so we should expect the associated $\Phi$ value to be. All of this, I suspect, is also true (to a greater or lesser, but nonzero, extent) for any pair of distinct colours that we can consciously distinguish.

One possible response is that, even if not identical, the relevant $\Phi$ values must be close. However, this is too vague. The $\Phi$ values may be close in the sense that the difference between them is very small compared to the range of $\Phi$ values that human brains can produce, but still distinct enough to imply a swift (compared to human perception times) collapse in models consistent with all available empirical data. To confirm or exclude this possibility, we would need a quantitative estimate, presumably calculated from IIT applied to a (presently unavailable) neuron-level model emulating the observer's brain, together with quantitative parameter bounds for $\Phi$ -collapse models, in order to calculate the possible range of collapse rates for the green-blue superposed subject.

It is helpful to compare the case of proposals for gravitationally induced collapse $[19,20]$. These suggest that collapses of superpositions of matter states with distinct gravitational fields take place very fast, even when the relevant fields are almost identical by ordinary laboratory scale measures. On Diosi's and Penrose's estimates, a superposition of distinct mass distributions should collapse long before the relevant gravitational fields could be directly distinguished by any laboratory measurement, for example. It seems quite natural to imagine that almost imperceptible differences in levels of consciousness could similarly cause swift collapse in viable models.

In fact, experimental evidence [21] recently appears to have excluded the most natural proposal [19] (so far) for a quantitative estimate of the rate of gravitationally induced collapse. This only reinforces the point that we need parametrised models and empirical bounds to draw clear conclusions about viable consciousness-collapse models, just as for any other form of dynamical collapse model. It should be noted that Diosi, the other authors of Ref. [21] and Penrose all continue to find the hypothesis of gravitationally induced collapse attractive and natural, while accepting that it may require a different formulation. Whether or not it will ultimately prove theoretically or empirically justifiable, the intuition remains that collapse should be swiftly induced for superpositions of states whose associated gravitational fields are very similar by any directly observable measure. If, as Chalmers-McQueen argue [2], consciousness-induced collapse models deserve to be on the table at all, the analogous intuition for these models seems similarly natural. Of course, it may be refutable (possibly even from existing data) for IIT-based or other specific collapse models, but I see no reason to dismiss it without quantitative arguments.

Another possible response is that, while the states of observing blue screen and green screen may produce different $\Phi$ values, we could tune them to produce two states with the same $\Phi$ value. The thought here is that $\Phi$ should (at least to very 
good approximation) vary continuously with controllable parameters such as screen size or intensity. So, if, say, the blue state has higher $\Phi$ value than the green state, we could intensify or enlarge the green screen, until the $\Phi$ values are equal. Supposing this is correct, one problem is that we still would not know which amplified green state has the same $\Phi$ value as the blue state, and so we would not be able to knowingly create a superposition state-again, unless we had a neuron-level model emulating the observer's brain and allowing us to calculate directly the relevant $\Phi$ values. We could try a range of amplifications, in the hope that one of them is right-but again, without quantitative calculations and quantitative models we cannot be sure any given set has significant probability of producing a superposition of states with identical $\Phi$ values, or of states with close enough $\Phi$ values to produce a long-lived superposition. We could turn a dial continuously, amplifying the green screen from much below the intensity of the blue screen to much above. At some instant, one might think this should produce equal $\Phi$ values. One problem with this is that $\Phi$ depends on network transitions, and when a dynamic image's trajectory includes a given image, the $\Phi$ value of the former may not necessarily be close to that created by a static version of the latter. Another is that the argument needs a superposition of states whose $\Phi$ values remain equal for a significant length of time.

In any case, we still have the problem of $\Phi$ instability arising from instability of the content of consciousness, because of the vagaries of the human brain. Even if one of these methods produces a superposition of identical $\Phi$ states, for suitably tuned blue and green screen observations, at a given point in time, the states will not persist as pure perception states and their $\Phi$ values should not be expected to remain identical for any significant time.

\section{Ideal Observers}

What, though, about computers or other artificial observers? While we may not have very strong reasons to assume that they have conscious states, IIT-which can assign high values of $\Phi$ even to quite simple computing devices-suggests that they do. Perhaps this is true of any plausible theory that quantifies consciousness as a function of information flow in networks. We can certainly design abstract networks that behave in precisely similar ways in response to different inputs. For example, we could make a network with a detector array whose individual detectors generate a 0 every second if they receive blue light above a threshold intensity and a 1 if they receive green light above that intensity, and then send these signals to two separate identical sub-networks that process them and characterise the blue or green shape detected. IIT suggests a suitably designed network of this type would be conscious for either input, with the same value of $\Phi$ in each case. However, IIT postulates [22] identical conscious states associated with isomorphic networks. ${ }^{2}$ A superposition state of the network observing blue and green screens would not collapse, but both components would be associated with the same conscious state (whatever it is).

\footnotetext{
${ }^{2}$ I thank Kelvin McQueen for helpful discussions on this.
} 
Still, it is surely possible to find networks that produce precisely the same value of $\Phi$ in two different non-isomorphic states, to which IIT would assign distinct conscious states. A $\Phi$-collapse model would predict that such a network could be put into a persistent superposition of conscious states. Is this a good reason to reject $\Phi$ -collapse models?

To argue that it is, one has to assume that persistent superpositions of distinct conscious states are generally unacceptable, even in cases very far from our own experience. The thought here would presumably be that such states are just nonsensical or uninterpretable, or at least that it is eminently reasonable to postulate some fundamental principle that excludes them, perhaps following a loose analogy with Penrose and Diosi's suggestion that nature does not allow superpositions of distinct spacetimes.

One might try to argue that if any persistent superpositions of distinct conscious states were straightforwardly intelligible, then purely unitary quantum theory would be straightforwardly intelligble, and there would be no motivation to consider any form of collapse. Arguments like this (e.g. [23, 24]) are used to justify lower bounds on collapse rates in dynamical collapse models, but in that context they apply only to humans, relying on our introspective impression that we quickly see definite outcomes to a priori uncertain quantum experiments. To run a more general version of the argument one would need to show that any sensible interpretation of persistent superpositions of distinct conscious states for general (not necessarily human) observers would necessarily explain the appearance of single worlds governed by Born rule probabilities for human observers in purely unitary quantum theory.

However, there are other possibilities that seem logically coherent. For example, one could imagine a psychophysical principle according to which consciousness disappears in any persistent significant superposition of states that, individually, would correspond to distinct conscious states. (Here "significant" is a placeholder for some quantitative criterion.) On such a rule, our ideal network would "be in conscious state $A$ " or "be in conscious state $B$ " given the corresponding inputs, but have no experience when given a superposed input. If humans cannot sustain persistent superpositions of equal- $\Phi$ states (even in the absence of any collapse postulate) because the $\Phi$ values of our complex and noisy brains continually vary whatever their initial state, we would never have encountered this effect. Even if we managed to sustain a superposition momentarily, it would swiftly become a superposition of unequal- $\Phi$ states and collapse. We should expect this to leave no memory of the effect, for at least two reasons. First, the effect may be so fleeting as to be almost imperceptible. Second, we are assuming some form of extension of IIT's model of consciousness, according to which brain states cause conscious states but there is no independent causal effect in the other direction. Given this, any post-collapse memories are defined by the post-collapse brain state, and so would be of definite conscious states corresponding to one or other component of the superposition.

It seems then that, to preclude superpositions of equal- $\Phi$ states for artificial devices, we simply have to assume (a) that a device in a superposition of such states must necessarily be conscious, (b) there is no conceivable sensible account of what its conscious state could be that does not undercut the motivation for considering collapse models at all. These aren't ridiculous assumptions-a thought underlying 
(a) might be that if individual states carry consciousness then any superposition should (perhaps because of some loose analogy with charge or mass, or because "being conscious" should behave like a binary quantum observable) and a thought underlying (b) might be that talking about a superposition of conscious states is just a category error-but they don't seem completely compelling.

We should note too that there might turn out to be interesting theories of consciousness that (unlike IIT) assign consciousness only to biological systems. For any such theories, there are no ideal observers, and so no way to create equal- $\Phi$ states. More generally, this is true for any theories whose measure for all physical systems has similar features to IIT's $\Phi$ for biological systems, in that it is always unstable and will have different time evolutions in different states where it is initially equal.

\section{Qualia and Symmetries}

It is worth considering more carefully the intuition that humans are (at least to very good approximation) equally conscious viewing different coloured screens in an otherwise darkened room. There is a weak version of this intuition: in either case the human is awake, and all waking conscious states should have very similar levels of $\Phi$. If one only holds this weak version, then the argument has just as much force if we consider even radically qualitatively different waking states_-viewing the blue screen, stroking a hamster, listening to Bach, and so on. The complex definition of $\Phi$ in IIT suggests, though, that radically different waking states should generally have different values of $\Phi$. If IIT is correct and our introspective intuitions also are, the differences should be smaller than the difference between any of them and a drowsy or dreamless sleeping state. It may also be hard to predict, from introspection, how the $\Phi$ levels of the waking states are ordered. Still, a $\Phi$-collapse will predict that superpositions of these states collapse, perhaps (depending on the quantitative details) very swiftly. If it were possible to produce a "cleaned-up" artificial human emulator that can sustain stable $\Phi$ values and can reproduce pure versions (without the complicating associations) of the human experiences of viewing a blue screen, stroking a hamster and listening to Bach, we should still expect the $\Phi$ levels to be different and so still expect $\Phi$-collapse. The weak version of the intuition thus does not seem able to support Chalmers-McQueen's argument, even for idealized human emulators.

It seems to me that the argument feels initially plausible because it relies on a stronger version of the intuition: that watching a blue screen and a green screen are qualitatively identical, up to interchanging the colours, and that images of the same area and intensity in different colours should evoke the same $\Phi$. This in turn seems to rely on the intuition that there is a natural relation-a colour flip map-between the conscious states evoked. However they are represented mathematically, they have the same structure: a shape or a collection of pixels of the same colour. For example, if consciousness is a collection of qualia, we can map the blue-screen conscious state to the green-screen one simply by replacing every blue quale with a green quale, and vice versa. If consciousness has some other structure, we can find an analogous map with the same effect. While the complexities of human brains 
may make the stronger intuition not quite precisely correct for us, this intuition suggests hat the symmetries should be exact rather than approximate for an ideal (artificial) observer that is able to have stable and pure colour experiences.

In any theory of consciousness that maps physical states to mental states, this intuition seems to require an associated map acting on the physical brain states, which also defines an (approximate) symmetry of physical states. One way to see this is to ask what we would conclude if we learned that observing blue and green screens used radically different and unrelated brain pathways. I think we would come to doubt not only that the mind states are associated with similar levels of consciousness, but also that they are as closely related as introspection seems to suggest.

We can flesh all this out as postulating a commutative diagram:

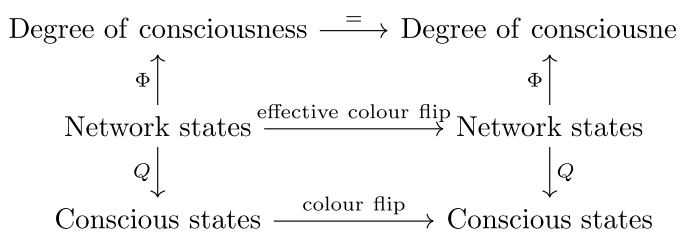

Here $Q$ is the map that characterizes the content and structure of any consciousness associated to any given information processing network: in IIT it is supposed to be representable mathematically by the so-called qualia shape or Q-shape. The "effective colour flip" on network states maps a network state that evokes one colour to a network state that evokes another; that is, it has the effect of flipping the colour evoked in the conscious state.

Why would one expect an effective colour flip map on network states to give the equality in the $\Phi$ values in the top line? The third line motivates this, but to justify it we need to consider the properties of the network model and the hypothesized map $\Phi$. In the concrete example of IIT, a network $\times_{i} A_{i}=A_{1} \times \ldots \times A_{n}$ begins at time 0 in some initial state $a_{1}(0) \times \ldots \times a_{n}(0)$, and at each subsequent time step $t$ its state $a_{1}(t) \times \ldots \times a_{n}(t)$ depends (in general probabilistically) on the previous state according to specified causal rules, which for fixed networks are taken to be timeindependent. The degree of consciousness $\Phi(t)$ at time $t$ is determined by the state at time $t$ and the rules determining the state at time $t+1$. The intuition that there is an effective colour flip map is mathematically natural (i.e. does not require fine-tuned coincidences) only if there is an associated permutation symmetry $\rho$ such that the network $\times_{i} A_{i}$ in initial state $\times_{i} a_{i}(0)$ is equivalent to the network $\times_{i} A_{\rho(i)}$ in initial state $\times_{i} a_{\rho(i)}(0)$, in the sense that the causal rules map isomorphically, so that the probability of any future state $\times_{i} a_{i}(t)$ in the former equals that of $\times_{i} a_{\rho(i)}(t)$ in the latter. This is also the natural physical justification: blue and green screens produce different inputs whose processing paths through the network are isomorphic.

But then the intuition fails: IIT does not assign different conscious states to isomorphic network states [22]. Moreover, this is not a peculiar feature of IIT: it seems natural for any theory mapping networks to conscious states. If the symmetry is precise, what could there be about $\times_{i} A_{\rho(i)}$ that could give it the conscious state of greenscreen perception where $\times_{i} A_{i}$ gives blue? The only difference is in the physical locations of the relevant network components. It seems very unnatural to suppose that 
conscious qualia depend on these at all, and even more unnatural to hypothesize (as one would have to) some fixed map from the network's location in configuration space to colour perception space. Any choice of such a map would be completely arbitrary: why should, say, a translation $1 \mathrm{~mm}$ to the left swap blue with green? And any permutation of colour qualia would appear to define an equally valid and equally (im)plausible map.

It might be argued that a precisely symmetric network is never physically realisable. However carefully one tries to build a symmetric network, its transition probabilities will always vary slightly from the designed specifications, in a way that breaks the symmetry. Indeed, but if so, the corresponding $\Phi$ values should also differ at least slightly, and we return to the discussion of the previous section.

It might also be noted that, even if a physical network is symmetric in its highlevel behaviour (as encoded in the network transition probabilities), its components will never be identical. So, they can always be distinguished by features other than their location. Again (within classical models, and so ignoring the possibility of and also the issues raised by indistinguishable quantum states) this is true. But if these features are irrelevant to the conscious state then they do not matter; if they are relevant, then the network model of consciousness considered was inadequate, and a deeper model including these features is needed. In this last case, again, the values of $\Phi^{\prime}$ (the measure of consciousness in the new model) should differ.

\section{Symmetries and Mind States}

There is an intriguing general point underlying this argument. Discussions of consciousness are often framed in a way that seems implicitly to appeal to some form of symmetry among conscious states, or at least leaves open the thoughts (i) that there may be such a symmetry and (ii) this makes the arguments more plausible. For example, James' discussion [25] of the difficulty in understanding the evolutionary emergence of epiphenomenal consciousness given the strong correlations between pleasure (pain) and evolutionary (dis)advantage looks cleanest if one can simply take pleasure and pain to be positive and negative values of a single scalar quantity, whose sign one could imagine being reversed. Arguments involving hypothetical beings whose consciousnesses are identical to ours except that they experience altered spectra (say with blue/green exchanged) similarly seem cleanest if colour sensations are not only considered as elementary components of mind states, independent of any other qualia, but also thought of as completely interchangeable.

Introspection gets us only so far on this. Certainly colour sensations feel as though they belong to a common class, different from auditory sensations, or verbalised thoughts, or emotions (though they may evoke at least the last two). But is it possible to experience pure colour and nothing else? For the reasons given earlier, I'm not so sure. And do elementary pure colour sensations even exist (whether or not they can be experienced apart from other qualia)? It feels hard to me to identify distinct components of colour sensations, even when experiencing colours that are mixtures of primary colours. But that doesn't seem to be a strong argument that colour sensations - even primary colour sensations - aren't fundamentally composite. 
Is exchanging blue and green qualia more analogous to exchanging up and down quarks (an approximate symmetry) or positive and negative charges (a better approximate symmetry) or applying the PCT operator (an exact symmetry) in the material world? Or is it more like exchanging $\mathrm{CO}_{2}$ and $\mathrm{N}_{2} \mathrm{O}$ - an operation which might look superficially like a symmetry, if one does not know the underlying structure of the world, but is not one in any meaningful sense? Introspection doesn't seem to give clear answers.

Are different colour perceptions evoked by near-isomorphic brain states? I don't think we know this either. Even if they are, the deviations of the states from isomorphism, although small, may be crucial rather than incidental to the experiences evoked. So our knowledge about our own physical brain states also does not seem to offer strong support for either a physical symmetry (between brain states associated with different colour qualia) or a mental one (between different colour qualia).

The case for a pain/pleasure symmetry seems weaker still. A pure colour sensation at least seems (if perhaps mistakenly) imaginable. It seems much harder to imagine a pure pain or pleasure sensation, untethered to any past or anticipated event, to any more complex emotion, or to any region of the body. I am also not sure that it makes sense to describe a pain as a negative pleasure; pains and pleasures (and even different types of pain and pleasure) feel qualitatively different, not quantitatively. It's true that rational human behaviour is often modelled as an attempt to maximize a scalar quantity, utility, that can take positive and negative values. But theoretical justifications for this (e.g. [26]) come from plausible axioms about what constitutes rational behaviour. They do not require the hypothesis that our experiences can be qualitatively characterised by a utility measure.

Finally, as noted above, the hypothesis that there are isomorphic states of an idealised brain that evoke distinct pure colour qualia related by a symmetry is quite problematic. If we postulate the symmetry, it should apply both to brain states (those associated with pure colour qualia) and mind states (those experiencing pure colour qualia). But we then need to break the symmetry again in order to get a map from specific brain states (associated with some specific colour qualia) to specific mind states (the experience of those specific qualia). This is not inconceivable in principle - spontaneous symmetry breaking is a familiar phenomenon elsewhere in physics. But spontaneous symmetry breaking needs an explanation-for example, dynamics induced by a potential with a degenerate ground state-and we have no substantive model here that could offer one. It would seem rather perversely baroque to postulate symmetries in both the material and mental worlds, for which we have no good evidence, and then further suggest that some unknown symmetry breaking mechanism in some unknown model explains how their actions come to be related by maps with the properties given in (1), with the symmetry realised in both worlds but broken by $Q$. Again, similar comments apply to models involving isomorphic pleasure/pain states.

In both cases, then, I suggest that the hypothetical symmetries cannot naturally be accommodated within physical theories of consciousness that aim to map brain states to mind states. IIT usefully illustrates this, but the argument is independent of the details of IIT. If so, arguments that both assume some such theory is correct and appear to be implicitly drawing some strength from a hypothetical symmetry need to 
be carefully examined, and if possible reframed in a way that is explicitly independent of symmetry hypotheses..$^{3}$

\section{Earlier Relevant Work}

After circulating the first draft of this paper, my attention was drawn to earlier work on symmetries and consciousness, including intriguing discussions by Hurley [27], Lee [28] and Chalmers[29]. Each of these examines the case of left-right symmetry, which perhaps illustrates mostly clearly the points at issue here, and Lee's discussion [28] is particularly relevant.

This topic deserves an independent discussion, which I hope to give elsewhere. Here I just summarize the implications of the arguments above. Suppose consciousness is described by a theory like IIT, which defines a mathematical map from a space of classical descriptions of brain/network states to the space of associated mind/conscious states. Consider a brain/network with perfect left-right symmetry. If the map associated distinct, symmetrically related brain/network states to distinct mind/conscious states, it would effectively give an intrinsic label on three dimensional coordinate systems, distinguishing left-handed sets of coordinates from righthanded. While that is logically possible, it would be surprising, since it would break a symmetry of classical physics. (Even in a model based on quantum states, it would be surprising, since we know no reason why the violations of parity in quantum field theory should be relevant to consciousness.)

The more natural possibility, we have argued, is that symmetrically related brain/ network states are associated to identical conscious states. A hypothetical creature with a perfectly left-right symmetric neural network would not distinguish "leftish" and "rightish" sensations, although it might respond appropriately (and differently, though symmetrically) to stimuli on the left and right. Approximately left-right symmetric creatures may distinguish between perception or proprioception qualia associated with events on their left and right. These may perhaps feel to them (as they do for us) qualitatively similar and it may seem intuitively plausible to them (as it perhaps does to us) that they are in some sense related. But, on the view we are

\footnotetext{
3 James argues against an epiphenomenal theory of consciousness, which in my view describes the most natural interpretation of IIT when taken as a fundamental theory. He does not discuss a brain-mind map in detail, nor suggest how a scientific theory of the evolution of consciousness could overcome his argument. The relevance of our arguments to his discussion is thus contingent on the form of the unspecified theory. In any case, my hunch is that his argument could be adequately reframed: a much more careful discussion is needed, but here is a sketch. I find it hard to accept that it is simply tautologous to say that activities good or bad for our genetic survival are respectively pleasurable or painful. If we agree that the statements are more than tautologies then we agree, I think, that in the present state of our understanding we could imagine the world being otherwise. That is, the correlations with evolutionary (dis)advantage need some explanation, which we presently do not have. There is an obvious naive explanation-we have evolved minds and bodies interacting so that our physical behaviour is generally pleasure-seeking and pain-avoidant-but it is incompatible with epiphenomenalism. This was James' essential point, and we do not need to imagine a simple pleasure/pain symmetry defining a swap map to run this framing of the argument.
} 
arguing for, this intuition cannot be made precise. The states are not approximations to distinct but perfectly symmetrically related qualia states. Structural asymmetry is crucial to the sense of left-right distinctness. Eliminating the asymmetry (for example, by some continuous deformation of the neural networks, if that were possible) would also eliminate the sense of distinctness.

\section{Summary}

We have argued that collapse models based on a single measure of consciousness seem consistent with experience. Thought experiments that attempt to place humans in superpositions of distinct but equally conscious states do not refute such models, because it seems unlikely that humans can sustain precisely the same level of consciousness in any state for long, or that there are distinct human conscious states that remain precisely equally conscious for long. Thought experiments involving artificial networks, which might-according to some theories of consciousness-sustain distinct and equally conscious states for long periods, do not refute the models either, since we do not know what, if anything, these networks would experience in superposition.

Another argument that single-measure collapse models are plausibly consistent with our experience has been suggested by Okon and Sebastian [7, 8], who discuss the effect of decoherence (of the measurement device) on the environment and hence on an observer's consciousness. In response, Chalmers and McQueen [2] argue that any effects of decoherence are screened off from the observer's consciousness in the blue- and green-screen experiment: they see only the screen. One could press this further by arranging quantum experiments in which blue or green light pulses are sent towards the observer's retina, without any classical amplification. The discussion can be made clearer still by using the type of ideal artificial observers considered above. Within an IIT model, these can easily be designed so that only the experimental outcome affects their information processing network, rendering any decoherence effects irrelevant. For these reasons, we believe our independent arguments are needed.

We have also argued that there are strong reasons to doubt the intuition that we can, from introspection, identify experiences that are likely to have equal measures on consciousness, according to some sensible theory mapping physical states to mental states. It relies implicitly on a notion of symmetry between distinct mental states, which is hard to make natural in such a theory. On the one hand, a symmetry between mental states ought to be associated with a symmetry between the associated physical states. On the other, physical states related by simple symmetries seem naturally associated with identical mental states.

We are not suggesting here that there is no motivation to consider collapse models based on a detailed (multi-parameter) mathematical description of the contents of consciousness. There are other reasons $[2,3]$ why one might prefer such models: for example, that this may give consciousness a causal role in nature that aligns with some intuitions. These are interesting lines of thought, but beyond our scope here. 
Acknowledgements This work was supported by an FQXi Grant and by Perimeter Institute for Theoretical Physics. Research at Perimeter Institute is supported by the Government of Canada through Industry Canada and by the Province of Ontario through the Ministry of Research and Innovation. I thank David Chalmers and Kelvin McQueen for circulating a draft of their article [2] and for very helpful and enjoyable discussions, and Johannes Kleiner for helpful comments on a draft.

Open Access This article is licensed under a Creative Commons Attribution 4.0 International License, which permits use, sharing, adaptation, distribution and reproduction in any medium or format, as long as you give appropriate credit to the original author(s) and the source, provide a link to the Creative Commons licence, and indicate if changes were made. The images or other third party material in this article are included in the article's Creative Commons licence, unless indicated otherwise in a credit line to the material. If material is not included in the article's Creative Commons licence and your intended use is not permitted by statutory regulation or exceeds the permitted use, you will need to obtain permission directly from the copyright holder. To view a copy of this licence, visit http://creativecommons.org/ licenses/by/4.0/.

\section{References}

1. Wigner, E.P.: Remarks on the mind-body problem. In: Good, I.J. (ed.) The Scientist Speculates. Heineman, Washington (1961)

2. Chalmers, D., McQueen, K.: Consciousness and the collapse of the wave function. In: Gao, S. (ed.) Consciousness and Quantum Mechanics. Oxford University Press, Oxford (2021)

3. Chalmers, D., McQueen, K.: Consciousness and the collapse of the wave function: Presentations. http://consc.net/qm/

4. Chalmers, D.: Dirty secrets of consciousness. Talk at FQXi 5th International Conference, Banff, August 2016, (2016)

5. Kremnizer, K., Ranchin, A.: Integrated information-induced quantum collapse. Found. Phys. 45(8), 889-899 (2015)

6. Okón, E., Sebastián, M.A.: How to back up or refute quantum theories of consciousness. Mind Matter 14(1), 25-49 (2016)

7. Okon, E., Sebastián, M.: A consciousness-based quantum objective collapse model. Synthese, pp. $1-21,(2018)$

8. Okón, E., Sebastián, M.A.: The subjective-objective collapse model: Virtues and challenges. In: Gao, S. (ed.) Consciousness and Quantum Mechanics. Oxford University Press, Oxford (2021)

9. Oizumi, M., Albantakis, L., Tononi, G.: From the phenomenology to the mechanisms of consciousness: integrated information theory 3.0. PLoS Comput. Biol. 10(5), e1003588 (2014)

10. Ghirardi, G.C., Rimini, A., Weber, T.: Unified dynamics for microscopic and macroscopic systems. Phys. Rev. D 34(2), 470 (1986)

11. Ghirardi, G.C., Pearle, P., Rimini, A.: Markov processes in Hilbert space and continuous spontaneous localization of systems of identical particles. Phys. Rev. A 42(1), 78 (1990)

12. Saunders, S., Barrett, J., Kent, A., Wallace, D.: Many Worlds?: Everett, Quantum Theory, \& Reality. OUP, Oxford (2010)

13. Dennett, Daniel C.: Consciousness explained. Penguin UK, (1993)

14. Barrett, A. B.: An integration of integrated information theory with fundamental physics. Front. Psychol. 5, 63 (2014)

15. Aaronson, S.: Why i am not an integrated information theorist (or, the unconscious expander). URL http://www.scottaaronson.com/blog/?p=1799

16. Cerullo, M.A.: The problem with Phi: a critique of integrated information theory. PLoS Comput. Biol. 11(9), e1004286 (2015)

17. Barrett, A.B., Mediano, P.A.M.: The Phi measure of integrated information is not well-defined for general physical systems. J. Conscious. Stud. 26(1-2), 11-20 (2019)

18. Chalmers, D., McQueen, K.: private communications. b

19. Penrose, R.: On gravity's role in quantum state reduction. General Relativity and Gravitation 28(5), 581-600 (1996) 
20. Diosi, L.: A universal master equation for the gravitational violation of quantum mechanics. Phys. Lett. A 120(8), 377-381 (1987)

21. Donadi, S., Piscicchia, K., Curceanu, C., Diosi, L., Laubenstein, M., Bassi, A.: Underground test of gravity-related wave function collapse. Nat. Phys. (2020). https://doi.org/10.1038/ s41567-020-1008-4

22. Balduzzi, D., Tononi, G.: Qualia: the geometry of integrated information. PLoS Comput. Biol. 5(8), e1000462 (2009)

23. Bassi, A., Deckert, D.-A., Ferialdi, L.: Breaking quantum linearity: Constraints from human perception and cosmological implications. EPL (Europhysics Letters) 92(5), 50006 (2010)

24. Kent, A.: Perception constraints on mass-dependent spontaneous localization. In S. Gao, editor, Consciousness and Quantum Mechanics. Oxford University Press, (2021). arXiv.org:1806.10396

25. James, W.: Are we automata? Mind 4, 1-22 (1879)

26. Savage, L.J.: The theory of statistical decision. J. Am. Stat. Assoc. 46(253), 55-67 (1951)

27. Hurley, S.L.: Consciousness in Action. Harvard University Press, Cambridge (1998)

28. Lee, G.: The experience of left and right. pp. 291-315. (2006)

29. Chalmers, D.: Three puzzles about spatial experience. pp. 109-137. (2019)

Publisher's Note Springer Nature remains neutral with regard to jurisdictional claims in published maps and institutional affiliations. 